\title{
Pneumonia in Primary Antibody Deficiency Patients Receiving Immunoglobulin Therapy, Risk Factors, and Role of Prophylactic Antibiotics: Data From the US Immunodeficiency Network (USIDNET)
}

\section{Maha N. Syed}

The William T Shearer Center for Human Immunobiology at Texas Children's Hospital

\section{Carleigh Kutac}

Baylor College of Medicine

Jennifer M Miller

Baylor College of Medicine

\section{Rebecca Marsh}

University of Cincinnati

Charlotte Cunningham Rundles

Icahn School of Medicine at Mount Sinai, Division of Allergy and Clinical Immunology

Ramsay L Fuleihan

Columbia University Medical Center

Farrah Kheradmand

Baylor College of Medicine

Joud Hajjar ( $\nabla$ Joud.Hajjar@bcm.edu )

Baylor College of Medicine https://orcid.org/0000-0003-4633-1043

\section{Research Article}

Keywords: Specific antibody deficiency, common variable immunodeficiency, hypogammaglobulinemia, Agammaglobulinemia, upper respiratory infection, streptococcus pneumonia, Haemophilus influenzae, immunoglobulin replacement therapy

Posted Date: February 28th, 2022

DOI: https://doi.org/10.21203/rs.3.rs-1374483/v1

License: (c) (i) This work is licensed under a Creative Commons Attribution 4.0 International License. Read Full License 


\section{Abstract}

\section{Background}

Despite immunoglobulin replacement (IgRT) therapy, some patients with Primary Antibody Deficiency (PAD) continue to develop respiratory infections. Recurrent and severe respiratory infections, particularly pneumonia, can lead to significant morbidity and mortality. Therefore, we sought to determine the risk factors of developing pneumonia in PAD patients, already receiving IgRT.

\section{Methods}

We evaluated clinical, and laboratory features of PAD patients enrolled in the US Immune Deficiency Network (USIDNET) registry by April 2017. Patients were included if they met the following criteria: 1) PAD diagnosis (common variable immunodeficiency (CVID), Agammaglobulinemia, hypogammaglobinemia, and specific antibody deficiency (SAD). 2) Available data on infections before and after IgRT. Patients were excluded if they were not receiving IgRT, or no pre/post infections data were available. Descriptive and multivariable logistic regression analyses were used to identify factors associated with pneumonia post-lgRT.

\section{Results}

A total of 1286 patients met the inclusion criteria. Following IgRT, 226 patients (17.6\%) were reported to have at least one pneumonia episode. Using multivariate logistic regression analysis, we found a statistically significant increased risk of pneumonia in patients with asthma (OR: 1.79, 95\% Cl [1.20-2.67], $\mathrm{p}=0.004$ ), bronchiectasis (OR: $2.81,95 \% \mathrm{Cl}$ [1.64-4.80], $\mathrm{p}<0.001$ ), and interstitial lung disease (ILD) (OR: $2.90,95 \% \mathrm{Cl}[1.26-6.70], p=0.013)$. Patients who did not receive prophylactic antibiotics had a much higher risk of pneumonia (OR: $27.65,95 \% \mathrm{Cl}$ [8.63-88.59]; $p<0.001$ ), compared to those who received prophylactic antibiotics. For every 50 unit increase in IgA, the odds of reporting pneumonia post-lgRT decreased significantly (OR: $0.82,95 \% \mathrm{Cl}[0.69-0.97], \mathrm{p}=0.018$ ). Patients with X-Linked Agammaglobulinemia had a lower risk of pneumonia than other PAD diagnoses (OR: $0.84 ; 95 \% \mathrm{Cl}[0.75-$ 0.93]; $p=0.001)$. The infectious organism was reported in 39 of 226 patients who reported pneumonia after IgRT. Haemophilus influenzae was the most frequently reported $(n=11,28.21 \%)$, followed by Streptococcus Pneumoniae ( $\mathrm{n}=9,23.08 \%)$.

\section{Conclusion}

Our findings suggest a role for prophylactic antibiotics patients with PAD, especially in those with preexisting lung disease. However, our study is limited by the cross-sectional nature of the USIDNET database and limited longitudinal data. Therefore, prospective studies evaluating the indications of prophylactic antibiotics are warranted.

\section{Clinical Implications}


Patients with primary antibody deficiency and structural lung disease may benefit from prophylactic antibiotics to prevent persisted infections despite lgRT.

\section{Introduction}

Primary antibody deficiency disorders (PAD) are humoral immune deficits characterized by defective immunoglobulin production and function, recurrent respiratory, and gastrointestinal tract infections which significantly contribute to morbidity and mortality ${ }^{1-3}$. Recurrent and severe respiratory infections have been associated with poor quality of life in $\operatorname{PAD} 4,5$, and could lead to chronic, irreversible lung disease (e.g., bronchiectasis), ${ }^{6-9}$ even in those with subclinical infections ${ }^{10}$.

Treatment with immunoglobulin replacement therapy (IgRT) via intravenous (IV) or subcutaneous (SC) routes are used to reduce the incidence of infections ${ }^{11}$. However, a proportion of patients with PAD continue to have persistent respiratory infections ${ }^{12-14}$. There is a wide variation in the use of prophylactic antibiotics for patients with PAD, with some studies showing minimal efficacy for those patients already receiving $\lg \mathrm{RT}^{15,16}$.

Given the challenge and importance of reducing the risk of recurrent respiratory infection, particularly pneumonia, in patients with PAD, we aimed in this study to identify the pathogens responsible for pneumonia in patients with PAD after initiation of IgRT, and to explore factors that may predict risks to develop pneumonia after the initiation of IgRT.

\section{Methods}

\section{Data sources}

Data for analyses were acquired in a de-identified format from the US Immunodeficiency Network (USIDNET) registry, a research consortium established to advance scientific research on inborn errors of immunity. The study was approved by the USIDNET Steering Committee and the Baylor College of Medicine Institutional Review Board (IRB). The registry is populated by participating clinicianinvestigators at thirty-nine academic institutions in the United States and Canada. Data in the registry include demographic, clinical, and laboratory information abstracted and entered by the investigators and medical records submitted by participants, which are confirmed and entered by the investigator. In April 2017, we queried the USIDNET patient registry for demographic, clinical, laboratory data, including immunoglobulin levels, infectious pathogens, antibiotic therapy, and immunoglobulin therapies.

Patients were included if they had a confirmed PAD diagnosis of Common Variable Immune Disorder (CVID), Agammaglobulinemia, Specific Antibody Deficiency (SAD), Hyper-IgM Syndrome (HIGM), Hypogammaglobulinemia (unknown cause and no listed genetic defect). We excluded patients if they were not receiving IgRT, or pre/post infections data were unavailable.

\section{Statistical analysis}


Categorical variables were summarized using frequencies and percentages, and continuous measures were summarized using mean \pm standard deviation (SD), or median with interquartile ranges (IQRs).

Univariate comparisons were made using t-test, chi-square test, Fisher's exact test, and Wilcoxson's ranksum tests. Variables with a $\mathrm{p}<0.25$ were considered for inclusion in an exploratory multivariate logistic regression model.

A backward stepwise selection method was used to build the multivariable regression model. First, all variables were entered into a preliminary model, and were reviewed. Then, the highest p-value was eliminated, and the model was re-run. This was repeated at every step until all $p$-values were significant $(p<0.05)^{17}$.

Odds ratios (OR) and their corresponding 95\% confidence intervals (Cl) were calculated for each predictor to examine their contribution to the likelihood of having persistent respiratory infection after initiation of Ig RT. All data were analyzed using (Stata/IC, Version 12.11).

\section{Results}

\section{Demographics and clinical characteristics of the baseline cohort}

At the time of our query (April 2017), the USIDNET database included 2230 patients with PAD. Out of those, there were $\mathrm{N}=1286$ patients with a confirmed diagnosis of PAD who were treated with $\operatorname{lgRT}$ and had available information on infections post-lgRT. The mean age at PI diagnosis was 22.5 years $(0-84)$. Most patients (82.1\%) identified as Caucasian, and $33.7 \%$ were female. The most-reported diagnosis was CVID (67 .7\%). Complete patients' characteristics are summarized in Table 1s. Lung disease (32.7\%) was the most commonly reported comorbidity, including asthma (21.5\%) and bronchiectasis (7.54\%), followed by hematological autoimmune disease and gastrointestinal disease ( $26.1 \%$ and $19.6 \%$, respectively). Out of 1286 included in the study, 339 patients were reported to be receiving prophylactic antibiotics. Patients' comorbidities are further summarized in Table $1 \mathrm{~s}$. 


\section{Patients with Pneumonia Post lgG Therapy $(n=226)$}

Characteristic:

Gender, n (\%)

Female

$87 \quad 38.50 \%$

Male

$139 \quad 61.50 \%$

Age at PI Diagnosis (years)

All patients, median (range)

12

$(0.1-70)$

Females, median (range)

28

$(0.8-70)$

Males, median (range)

6.4

$(0.1-63)$

Time in Years from Symptom Onset to Diagnosis, median (range)

3

$(0-54.1)$

PAD Diagnosis, $\mathrm{n}(\%)$

CVID

$154 \quad 68.14 \%$

Hypogammaglobinemia (including XLA, BTK)

$54 \quad 23.89 \%$

Specific Antibody Deficiency

$7 \quad 3.10 \%$

Hyper-IgM (including CD40L)

$11 \quad 4.87 \%$

Race/ ethnicity, n (\%)

White or Caucasian

$167 \quad 73.89 \%$

Black or African American

$12 \quad 5.31 \%$

Asian or Pacific Islander

3

$1.33 \%$

Other or more than one race

3

$1.33 \%$

American Indian/Native American

1

$0.44 \%$

Unknown

40

$17.70 \%$

Route of IgRT, $n$ (\%)

Intravenous

$129 \quad 57.08 \%$

Subcutaneous

$57 \quad 25.22 \%$

Unknown

$39 \quad 17.26 \%$

Intramuscular

1

$0.44 \%$

Abbreviations: COPD: Chronic Obstructive Lung Disease ITP: Idiopathic Thrombocytopenia Purpura, 
GI: Gastrointestinal Disease, IBD: Inflammatory Bowel disease, EOE: Eosinophilic Esophagitis, LFTs: Liver function test, PLE: Protein-losing enteropathy.

\section{Post-treatment pneumonia and causative pathogens}

Pneumonia was reported in 226 patients after the initiation of IgRT (Table 1). Males reported a higher post-IgRT pneumonia frequency compared to females (61.5\% vs. $38.5 \%)$. Females had a later age of diagnosis in this cohort with a median age of 28 years compared to median age of 6.5 years for males. A PAD diagnosis of CVID (68.1\%) was most reported, followed by Hypogammaglobinemia (23.9\%) (Table 1). Lung disease was the most common comorbidity (56.2\%) (Table 2). Thirty-nine out of 226 patients who reported pneumonia after IgRT also reported the organism of infection that caused their pneumonia. H. influenzae was the most frequently reported $(n=11,28.21 \%)$ followed by S. pneumoniae $(n=9,23.08 \%)$. 
Table 2

Patient comorbidities: Patients with

Pneumonia Post IgG Therapy $(\mathrm{n}=226)$

\begin{tabular}{|c|c|c|}
\hline \multicolumn{3}{|l|}{ Comorbidities, n (\%) } \\
\hline \multicolumn{3}{|l|}{ Any respiratory condition } \\
\hline Asthma & 74 & $32.74 \%$ \\
\hline Bronchiectasis & 38 & $16.81 \%$ \\
\hline COPD & 17 & $7.52 \%$ \\
\hline Interstitial Lung Disease & 12 & $5.31 \%$ \\
\hline Granuloma & 8 & $3.54 \%$ \\
\hline Lung Disease & 127 & $56.19 \%$ \\
\hline Lung Nodules & 1 & $0.44 \%$ \\
\hline \multicolumn{3}{|l|}{ Other conditions } \\
\hline Autoimmune & 24 & $10.62 \%$ \\
\hline Hematological & 78 & $34.51 \%$ \\
\hline ITP & 17 & $7.52 \%$ \\
\hline GI & 48 & $21.24 \%$ \\
\hline Adenopathy & 28 & $12.39 \%$ \\
\hline Celiac Disease & 3 & $1.33 \%$ \\
\hline IBD & 18 & $7.96 \%$ \\
\hline Anemia & 41 & $18.14 \%$ \\
\hline Neutropenia & 17 & $7.52 \%$ \\
\hline Allergies & 34 & $15.04 \%$ \\
\hline Liver & 20 & $8.85 \%$ \\
\hline Spleen & 27 & $11.95 \%$ \\
\hline Abnormal LFTs & 6 & $2.65 \%$ \\
\hline EOE & 5 & $2.21 \%$ \\
\hline Liver Failure & 5 & $2.21 \%$ \\
\hline Pancytopenia & 1 & $0.44 \%$ \\
\hline PLE & 3 & $1.33 \%$ \\
\hline
\end{tabular}


Comorbidities, n (\%)

Thrombocytopenia

19

$8.41 \%$

\section{Immunoglobulin Levels}

We then evaluated the impact of immunoglobulin levels on post-IgRT infection. Of note, Ig levels were provided one-time value. There was no available data on when those values were reported in relation to IgRT. Patients' immunological data are summarized in Table 3. 
Table 3

Immune phenotype: Patients with Pneumonia Post IgG Therapy $(\mathrm{n}=226)$

\section{Immunoglobulins mg/dL, (range)}

IgA median (range)

CVID

Hyper-IgM (including CD40L)

Specific Antibody Deficiency

Hypogammaglobinemia (including XLA, BTK)

IgG median (range)* * IgG levels measures while on IgRT $(n=43)$

CVID

Hyper-IgM (including CD40L)

Specific Antibody Deficiency

Hypogammaglobinemia (including XLA, BTK)

IgM median (range)

CVID

Hyper-lgM (including CD40L)

Specific Antibody Deficiency

Hypogammaglobinemia (including XLA, BTK)

Lymphocyte Count (cells/uL), median (range)

CVID

Hyper-IgM (including CD40L)

Specific Antibody Deficiency

Hypogammaglobinemia (including XLA, BTK)
9

15

6.99

73

7

965

972.5

1020

1227.5

145

23.5

28.5

117

58.65

9

1839

1700

4363

1435.2

2800
(0-667)

(0-667)

$(0-97)$

(22-1467)

(109-1467)

1020

(1120-1335)

(22-1270)

(0-648)

(0-645)

(20-648)

(20-159)

(0-91.6)

(200-10098)

(200-7958)

(1670-9000)

(900-6539)

(700-10098)

Abbreviations: CD40L: CD40 Ligand; CVID: Common Variable Immunodeficiency; XLA: X-linked Agammaglobulinemia; BTK: Bruton Tyrosine Kinase; COPD: chronic obstructive pulmonary disease; Hyper-IgM: Hyper-IgM Syndrome; GLILD: granulomatous and lymphocytic interstitial lung disease GLILD; IM, Intramuscular; IV, intravenous; SC, subcutaneous, PAD (Primary Antibody Deficiency

There was a significant difference in the median IgM value between patients who reported pneumonia post-lgRT and patients who did not [23.5 vs. 29.0, $p=0.021]$. 
There was no significant difference between $\lg G$ or $\lg A$, median values between patients who developed pneumonia post IgRT compared to those who did not.

\section{Multivariate analysis for pneumonia after IgRT treatment in PAD patients}

To determine which variables to consider for building the multivariate logistic regression model for predicting pneumonia after beginning IgRT treatment, a univariate analysis was run, and variables with a $p<0.25$ were considered for the multivariate logistic regression model.

Variables with a p-value of less than 0.25 included: Age (18-26 vs. others), IgA ( $\mathrm{mg} / \mathrm{dl}-50$ unit increments), Absolute Lymphocyte Count (50-unit increments), Bronchiectasis, Interstitial Lung Disease, Autoimmunity, Hematological disease, Asthma, Allergies, Liver Disease, Splenomegaly, COPD, Adenopathy, eosinophilic esophagitis, IgRT therapy type (SCIG or IVIG only), immunosuppressives after beginning IgRT treatment, prophylactic antibiotics, and PID diagnosis (CVID vs. all others \& XLA vs. all others).

The backward stepwise selection method was used for the bimodal multivariate model. First, all variables were entered into a preliminary model, and the p-values were reviewed. Then, the highest p-value was eliminated, and the model was re-run. This continued at each step of the model until all p-values were considered significant $(p<0.05)$.

During the process of backward stepwise regression, the following variables were eliminated from the final model due to a p-value of larger than 0.05: Age (18-26 vs. others), Absolute Lymphocyte Count (50unit increments), autoimmunity, hematological disease, allergies, liver disease, splenomegaly, COPD, Adenopathy, EOE, IgRT therapy type (SCIG or IVIG only), taking immunosuppressives after beginning IgRT treatment, and PID diagnosis (CVID vs. all others).

After performing backward stepwise regression, the final multivariate model included Prophylactic Antibiotic use, XLA (vs. all PID diagnoses), Bronchiectasis, Asthma, Interstitial Lung Disease, and IgA $(\mathrm{mg} / \mathrm{dl})$. The area under the ROC curve was 0.7912 , indicating that our variable subset is a proper fit for predicting pneumonia after beginning IGRT treatment.

Below are the predicted outcomes for each variable (Table 4):

- Prophylactic Antibiotics

- Compared to patients who were on prophylactic antibiotics, the patients who were not on prophylaxis had an increase in the odds of reporting pneumonia after IGRT (OR: $27.65,95 \% \mathrm{Cl}$ [8.63-88.59]; $p<0.001)$, while holding all other variables constant.

- PID Diagnosis 
- When comparing all other PID diagnoses to patients who have the diagnosis of XLA, patients with the diagnosis of XLA had a decrease in the odds of reporting pneumonia after IGRT (OR: $0.84 ; 95 \% \mathrm{Cl}[0.75-0.93] ; \mathrm{p}=0.001$ ), while holding all other variables constant.

- Asthma

- Compared to patients who did not report asthma, patients who did report asthma had an increase in the odds of reporting pneumonia after IGRT (OR: 1.79, 95\% Cl [1.20-2.67], p =0.004), while holding all other variables constant.

- Bronchiectasis

- Compared to patients who did not report bronchiectasis, patients who did report bronchiectasis had an increase in the odds of reporting pneumonia after IGRT (OR: 2.81, 95\% CI [1.64-4.80], p< 0.001), while holding all other variables constant.

- Interstitial Lung Disease

- Compared to patients who did not report ILD, patients who did report ILD had an increase in the odds of reporting pneumonia after IGRT (OR: 2.90,95\% Cl [1.26-6.70], $p=0.013$ ), while holding all other variables constant.

- $\lg A(\mathrm{mg} / \mathrm{dL})$

- For every 50 unit increase in IgA, the odds of reporting pneumonia post-lgRT decreased significantly (OR: $0.82,95 \% \mathrm{Cl}[0.69-0.97], \mathrm{p}=0.018$ ).

Table 4

Factors that affect the risk of persistent infections post immunoglobulin replacement therapy

\begin{tabular}{|llc|}
\hline Determinants & Adjusted OR (95\%) & p-value \\
\hline Prophylactic Antibiotics (no vs yes) & $27.65(8.63-88.59)$ & $<0.001$ \\
\hline XLA (vs all other PID diagnoses) & $0.84(0.75-0.93)$ & 0.001 \\
\hline Comorbidity: ILD & $2.90(1.26-6.70)$ & 0.013 \\
\hline Comorbidity: Asthma & $1.79(1.20-2.67)$ & 0.004 \\
\hline Comorbidity: Bronchiectasis & $2.80(1.64-4.80)$ & $<0.001$ \\
\hline IgA (mg/dl) - 50-unit increments & $0.82(0.69-0.97)$ & 0.018 \\
\hline Area under the curve = 0.7912 & & \\
\hline $\begin{array}{l}\text { Abbreviations: XLA: X-linked Agammaglobulinemia ILD: Interstitial Lung Disease, OR: Odds ratio, Cl: } \\
\text { Confidence Interval }\end{array}$ & \\
\hline
\end{tabular}

\section{Discussion}


This exploratory analysis aimed to identify factors associated with pneumonia in patients with PAD following treatment with IgRT. Persistent respiratory symptoms and complications such as pneumonia pose a significant cause of morbidity and mortality among pediatric and adult patients with inborn errors of immunity ${ }^{18}$. However, its predictors are not well understood. As such, this study provides additional clarity to this challenging problem.

Consistent with previous literature, we have confirmed that Streptococcus pneumoniae and Haemophilus influenzae type b (hib) are the most common pathogens identified in patients with PAD with respiratory infections, ${ }^{19-21}$ even after IgRT. Although most commercially available IgRT products contain antibodies to Streptococcus pneumoniae and hib, the levels of those specific antibodies vary significantly between products $^{22,23}$, and preparations are not standardized for specific antibody content for the pathogens that most commonly cause infections in patients with inborn errors of immunity ${ }^{24}$. Additionally, the inability to directly transport IgG to mucosal membranes has been postulated to be a risk factor for persistent infections in patients with $\mathrm{PAD}^{16}$. Equally important, respiratory viral infections could lead to persistent and severe respiratory infections despite IgRT ${ }^{25}$.

From a host perspective, we have identified several significant predictors of pneumonia in patients with PAD after IgRT therapy. Consistent with previous studies, we noted that asthma, bronchiectasis, and interstitial lung disease significantly increased the risk of pneumonia in a patient with PAD despite receiving immunoglobulin replacement therapy ${ }^{26-29}$. Comorbidity of XLA and administration of IgA were in contrast protective. In CVID, the presence of bronchiectasis was associated with persistent respiratory infections despite lgRT in those with $\operatorname{lgA}$ level $<7 \mathrm{mg} / \mathrm{dL}$ and those with persistently lgG trough levels $<$ $400 \mathrm{mg} / \mathrm{dL})^{14}$, while, in XLA, bronchiectasis was the only predictor of increased risk of pneumonia following IgRT in the same center ${ }^{14}$.

We also found that patients on prophylactic antibiotics had a significantly lower risk of developing respiratory infection compared to those who did not receive prophylactic antibiotics. We have previously shown that prophylactic antibiotics are equally effective in preventing infections and hospitalization in specific antibody deficiency compared to immunoglobulin replacement therapy ${ }^{11}$. Lucas et al. showed a minimal benefit of prophylactic antibiotics ${ }^{15}$. However, the study only had 18 patients receiving prophylactic antibiotics for respiratory infections. Together, these data raise the need for controlled studies to identify the best approach to prevent persistent infections in patients receiving therapeutic doses of IgRT.

Further, we show that having a diagnosis of CVID, or SAD diagnosis, increased persistent pneumonia. CVID accounted for most patients, and most had chronic lung disease. We only included SAD patients receiving IgRT, thus resulting in a selection bias of more severe SAD cases in whom treating physicians identified a need for IGRT (rather than prophylactic antibiotics).

The main strengths of this study include the use of USIDNET registry data; its large size, prospective data collection from thirty-nine institutions, and the broad representation of PAD increases our ability to 
generalize results. To our knowledge, our study was the largest of its kind to evaluate predictors of respiratory infections in patients with PAD. While registries allow the collection of rare diseases, there are limitations in that reporting of cases is voluntary, and data might be entered once, or updated as per providers discretion. Hence, the frequency of infections/year could not be ascertained, as the USIDNet database does not allow for longitudinal study design. In addition, as this was a secondary analysis, we do not have access to patient charts, dosing of IgRT or its timing, and laboratory data such as IgG trough levels cannot be ascertained. Despite these limitations, our study provides insights on predictors for persistent infections in patients with PAD from both patient and pathogen-related factors.

\section{Conclusion}

IgRT significantly reduces but does not eliminate respiratory infections in patients with PAD. Having chronic lung disease, XLA diagnosis, and low IgA increase the risk of post-IgRT pneumonia, while receiving prophylactic antibiotics decreases the risk of post-IgRT pneumonia.

Our findings suggest a role for prophylactic antibiotics patients with PAD, especially in those with preexisting lung disease. Our study is limited by the cross-sectional nature of the USIDNET database and limited longitudinal data. Prospective studies evaluating the benefits of prophylactic antibiotics are warranted.

\section{Abbreviations}

AID Deficiency activation-induced cytidine deaminase deficiency

aOR Adjusted Odds Ratio

BTK Bruton's tyrosine kinase

CD40L CD40 Ligand

CVID Common Variable Immunodeficiency

$\mathrm{Cl}$ Confidence Interval

COPD Chronic obstructive pulmonary disease

GLILD Granuloma, interstitial lung disease

HIGM Hyper-IgM Syndrome

IG Immunoglobulin

IgRT Immunoglobulin Replacement Therapy 
IM Intramuscular

IQR Interquartile Range

IRB Institutional Review Board

IV Intravenous

IVIG Intravenous Immunoglobulin

OR Odds Ratio

PAD Primary Antibody Deficiency

SAD Specific Antibody Deficiency

SD Standard Deviation

SC Subcutaneous

SCIG Subcutaneous Immunoglobulin

TACI Deficiency Transmembrane activator and CAML interactor deficiency

USIDNET United States Immunodeficiency Network

XLA X-linked Agammaglobulinemia

\section{Declarations}

Ethical Approval: The study was approved by the USIDNET Steering Committee and the Baylor College of Medicine Institutional Review Board (IRB).

Consent to Participate: N/A

\section{Consent to Publish: N/A}

Authors Contributions CK and JH performed material preparation, data collection and analysis. The first draft of the manuscript was written by MS and JH. FK, JM and RM contributed critical review and comments on data analysis and manuscript endpoints and overall message. CCR, KS and RF provided significant contributions to the USIDNET database ( $>10 \%$ of patients included). All authors commented on previous versions of the manuscript. All authors contributed to the study conception and design. All authors read and approved the final manuscript.

Funding: This research project was funded by the US immune deficiency network research grant 
Competing Interest: Hajjar received grants from Immune Deficiency Foundation, the US immunodeficiency network, Chao-physician Scientist award, the Texas Medical Center Digestive Diseases Center, and the Jeffrey Modell Foundation. J. Hajjar received an honorarium/advisory from Horizon, Pharming, Baxalta, CSL Behring, the National guard, and Al-Faisal University Hospital outside the submitted work.

Availability of data and materials: The datasets generated during and/or analyzed during the current study are available from the corresponding author on reasonable request

Code availability: N/A

\section{References}

1. Wood P. Primary antibody deficiencies: recognition, clinical diagnosis and referral of patients. Clin Med (Lond) 2009; 9:595-9.

2. Hampson F, Chandra A, Screaton N, Condliffe A, Kumararatne D, Exley A, et al. Respiratory disease in common variable immunodeficiency and other primary immunodeficiency disorders. Clinical radiology 2012; 67:587-95.

3. Cunningham-Rundles $\mathrm{C}$, Bodian $\mathrm{C}$. Common variable immunodeficiency: clinical and immunological features of 248 patients. Clin Immunol 1999; 92:34-48.

4. Jiang F, Torgerson TR, Ayars AG. Health-related quality of life in patients with primary immunodeficiency disease. Allergy Asthma Clin Immunol 2015; 11:27.

5. Routes J, Costa-Carvalho BT, Grimbacher B, Paris K, Ochs HD, Filipovich A, et al. Health-Related Quality of Life and Health Resource Utilization in Patients with Primary Immunodeficiency Disease Prior to and Following 12 Months of Immunoglobulin G Treatment. J Clin Immunol 2016; 36:450-61.

6. Good RA, Mazzitello WF. Chest disease in patients with Agammaglobulinemia. Dis Chest 1956; 29:935.

7. Pasteur MC, Bilton D, Hill AT, British Thoracic Society Bronchiectasis non CFGG. British Thoracic Society guideline for non-CF bronchiectasis. Thorax 2010; 65 Suppl 1:i1-58.

8. Sweinberg SK, Wodell RA, Grodofsky MP, Greene JM, Conley ME. Retrospective analysis of the incidence of pulmonary disease in hypogammaglobulinemia. J Allergy Clin Immunol 1991; 88:96104.

9. Tarzi MD, Grigoriadou S, Carr SB, Kuitert LM, Longhurst HJ. Clinical immunology review series: An approach to the management of pulmonary disease in primary antibody deficiency. Clin Exp Immunol 2009; 155:147-55.

10. Kainulainen L, Varpula M, Liippo K, Svedstrom E, Nikoskelainen J, Ruuskanen O. Pulmonary abnormalities in patients with primary hypogammaglobulinemia. J Allergy Clin Immunol 1999; 104:1031-6. 
11. Joud H, Nguyen AL, Constantine G, Kutac C, Syed MN, Orange JS, et al. Prophylactic Antibiotics Versus Immunoglobulin Replacement in Specific Antibody Deficiency. Journal of Clinical Immunology 2019.

12. Busse PJ, Razvi S, Cunningham-Rundles C. Efficacy of intravenous immunoglobulin in the prevention of pneumonia in patients with common variable immunodeficiency. J Allergy Clin Immunol 2002; 109:1001-4.

13. Aghamohammadi A, Allahverdi A, Abolhassani H, Moazzami K, Alizadeh H, Gharagozlou M, et al. Comparison of pulmonary diseases in common variable immunodeficiency and X-linked agammaglobulinaemia. Respirology 2010; 15:289-95.

14. Quinti I, Soresina A, Guerra A, Rondelli R, Spadaro G, Agostini C, et al. Effectiveness of immunoglobulin replacement therapy on clinical outcome in patients with primary antibody deficiencies: results from a multicenter prospective cohort study. J Clin Immunol 2011; 31:315-22.

15. Lucas M, Lee M, Lortan J, Lopez-Granados E, Misbah S, Chapel H. Infection outcomes in patients with common variable immunodeficiency disorders: relationship to immunoglobulin therapy over 22 years. J Allergy Clin Immunol 2010; 125:1354-60 e4.

16. Kuruvilla M, de la Morena MT. Antibiotic prophylaxis in primary immune deficiency disorders. J Allergy Clin Immunol Pract 2013; 1:573-82.

17. Riffenburgh RH. Chapter 6 - Statistical Testing, Risks, and Odds in Medical Decisions. In: Riffenburgh $\mathrm{RH}$, editor. Statistics in Medicine (Second Edition). Burlington: Academic Press; 2006. p. 93-114.

18. Jesenak M, Banovcin P, Jesenakova B, Babusikova E. Pulmonary manifestations of primary immunodeficiency disorders in children. Front Pediatr 2014; 2:77.

19. Rezaei N, Aghamohammadi A, Siadat SD, Nejati M, Ahmadi H, Moin M, et al. Serum bactericidal antibody response to serogroup $\mathrm{C}$ polysaccharide meningococcal vaccination in children with primary antibody deficiencies. Vaccine 2007; 25:5308-14.

20. Aguilar C, Malphettes M, Donadieu J, Chandesris O, Coignard-Biehler H, Catherinot E, et al. Prevention of infections during primary immunodeficiency. Clin Infect Dis 2014; 59:1462-70.

21. Hermaszewski RA, Webster AD. Primary hypogammaglobulinaemia: a survey of clinical manifestations and complications. Q J Med 1993; 86:31-42.

22. Mikolajczyk MG, Concepcion NF, Wang T, Frazier D, Golding B, Frasch CE, et al. Characterization of antibodies to capsular polysaccharide antigens of Haemophilus influenzae type b and Streptococcus pneumoniae in human immune globulin intravenous preparations. Clin Diagn Lab Immunol 2004; 11:1158-64.

23. Lee S, Kim HW, Kim KH. Functional antibodies to Haemophilus influenzae type B, Neisseria meningitidis, and Streptococcus pneumoniae contained in intravenous immunoglobulin products. Transfusion 2017; 57:157-65.

24. Bonilla FA, Khan DA, Ballas ZK, Chinen J, Frank MM, Hsu JT, et al. Practice parameter for the diagnosis and management of primary immunodeficiency. J Allergy Clin Immunol 2015; 136:1186205.e1-78. 
25. Kainulainen L, Vuorinen T, Rantakokko-Jalava K, Osterback R, Ruuskanen O. Recurrent and persistent respiratory tract viral infections in patients with primary hypogammaglobulinemia. J Allergy Clin Immunol 2010; 126:120-6.

26. Lucas M, Lee M, Lortan J, Lopez-Granados E, Misbah S, Chapel H. Infection outcomes in patients with common variable immunodeficiency disorders: Relationship to immunoglobulin therapy over 22 years. Journal of Allergy and Clinical Immunology 2010; 125:1354-60.e4.

27. Brent J, Guzman D, Bangs C, Grimbacher B, Fayolle C, Huissoon A, et al. Clinical and laboratory correlates of lung disease and cancer in adults with idiopathic hypogammaglobulinaemia. Clinical \& Experimental Immunology 2016; 184:73-82.

28. Urm SH, Yun HD, Fenta YA, Yoo KH, Abraham RS, Hagan J, et al. Asthma and risk of selective IgA deficiency or common variable immunodeficiency: a population-based case-control study. Mayo Clin Proc 2013; 88:813-21.

29. Juhn YJ, Kita H, Yawn BP, Boyce TG, Yoo KH, McGree ME, et al. Increased risk of serious pneumococcal disease in patients with asthma. J Allergy Clin Immunol 2008; 122:719-23.

\section{Supplementary Files}

This is a list of supplementary files associated with this preprint. Click to download.

- Table1s.docx 